\title{
Markerless Alignment: Bridging the Gap Between Theory and Practice.
}

\author{
Christian Renken* and Bruce McEwen*.
}

${ }^{*}$ Resource for Visualization of Biological Complexity, Wadsworth Center, Albany, NY 12201

The ability to perform high through put tomography is at hand. Modern microscope stages are proving to be highly stable and reproducible. This has allowed automated data collection routines to acquire 120 image tilt series in under an hour![1] The bottle neck for real time tomographic reconstruction is now the alignment of images. Currently in most laboratories, alignment is attained by the use of fiducial markers, usually by depositing nano-meter gold beads on their sample. The position of the gold beads on every image is then used to calculated the proper transformation each image must undergo in order to align the set of images. Best alignment is achieved by selecting numerous gold beads in each image and ideally the same gold beads in every image. Automated routines that track beads in images after the user marks them in an initial image have proven robust, they still require editing. While this method of alignment gives optimal results, it is time consuming on the user, and limits the microscopists to image samples that have an adequate number of gold beads in the filed of view. Various groups have proposed methods of automation $[2,3]$ which have had limited success.

The fundamental challenge in automated methods is the noise inherent in tomographic data. In theory the method of cross correlation as described by Frank and McEwen [4] should correctly align a series of images. Unfortunately the presence of noise degrades the cross correlation function (ccf)(see figure 1). However given a reference with a high signal to noise ratio, cross correlation is extremely good at finding the reference in noisy data( figure 1).

Our algorithm cross correlates the low tilt angle images first, and then averages the aligned images to enhance the shared information. This average image is then used as the reference for the next increment of tilt images, which after cross-correlation alignment are averaged with the preceding images and a new reference is calculated. This has the effect of increasing the signal of the common lines in fourier space, and dampening the signal from the non common lines. Thus the reference image, while seemingly degraded (figure $2 \mathrm{a}$ ), increases in signal to noise ratio along the common line in fourier space (figure 2b). An added benefit is that because all aligned images are used for the reference image, any errors in alignment that may occur at higher tilt angles are not propagated [5].

References:

1. U. Ziese et al., J. Microscopy 205 (2002) 187.

2. D. Ress et al., J. Elec. Microscopy 48 (1999) 277.

3. S. Brandt et al., J. Struct. Bio. 136 (2001) 201.

4. J. Frank and B. McEwen in Electron Tomography, Plenum Press, New York, 1992

5. Supported by NIH RR01219 and NIH R01 GM6627001 


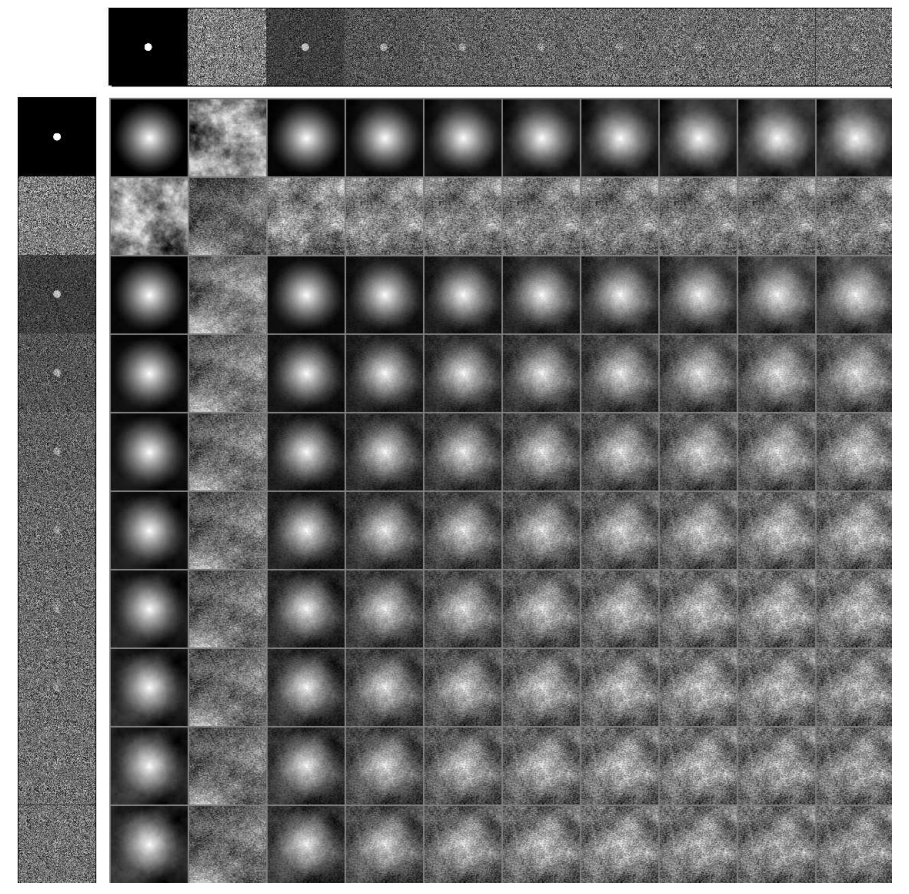

Figure 1: Matrix of cross correlation functions of an image (left column) with a reference (top row). First image/reference is the discrete signal. Second image/reference is complete random noise. Image/reference 3-10 decrease in signal to noise ratio. First column of the matrix demonstrates that even when the signal to noise ratio is small, the presence of a clean reference will provide for a strong ccf.

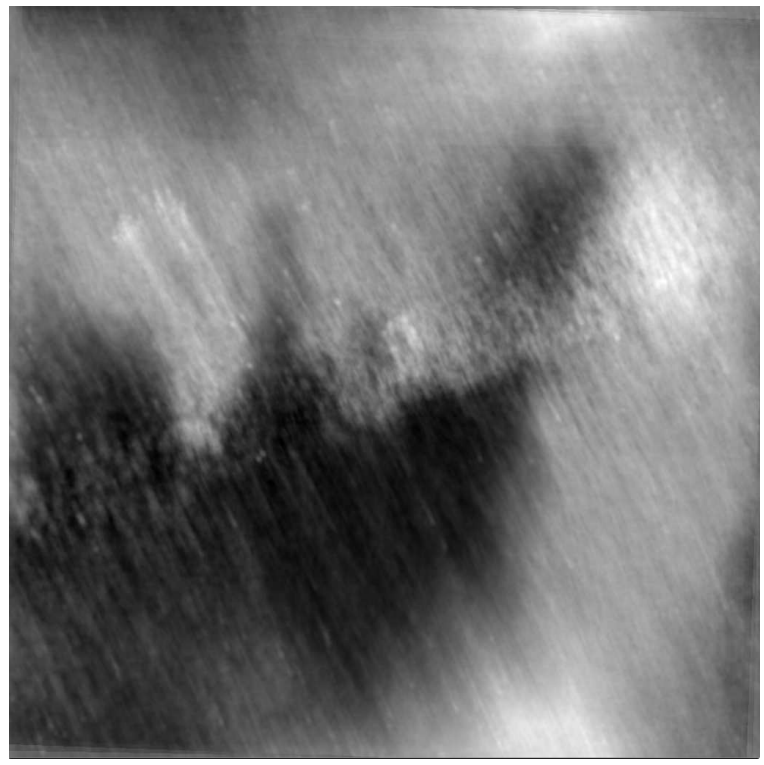

(a)

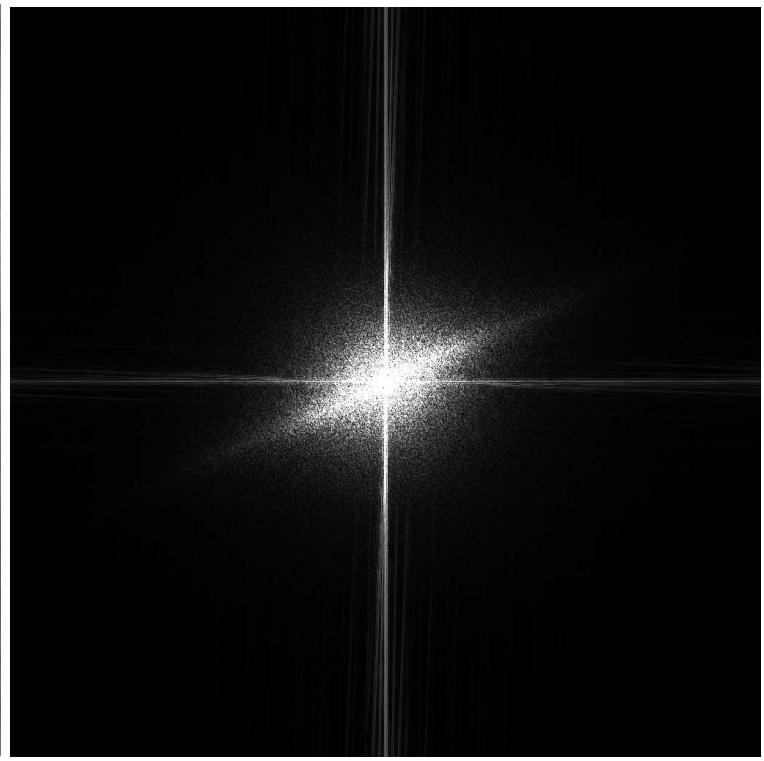

(b)

Figure 2: (a)Average of 75 aligned images from -60 to 60 taken with a cosine vary tilt scheme with a 2 degree interval at 0 degrees. (b) The Fourier transform of (a). 